\title{
Affective Responses of Early Life Photoperiod in Male Wistar Rats
}

\author{
Nassira Benabid, Ali Ouichou \\ Department of Biology, Faculty of Sciences, Ibn Tofail University, Kenitra, Morocco. \\ Email: benabidnassira@yahoo.fr
}

Received April 26 ${ }^{\text {th }}$ 2011; revised May 30 ${ }^{\text {th }}, 2011$; accepted July 28 ${ }^{\text {th }}, 2011$.

\begin{abstract}
Behavior changes season dependant are probably linked to change in day length or photoperiod. Although much research on seasonality in small mammals has focused on photoperiod manipulations in adults, early life photoperiod is also an important source of seasonal information and can establish individual's developmental trajectory by regulating somatic and reproductive development and affective responses to day lengths later in life. The experiments developed in this work are based on the hypothesis that early life photoperiod affect emotionality in adult rats. To cheek this hypothesis, male rats were exposed at birth to different photoperiods (LP: 16L/8D;SP: 8L/16D). 8, 16 or 24 weeks later, rats were subjected to different behavioral tests to quantify anxiety-like behavior. Independently of duration, rats exposed to SP exhibited higher levels of anxious-like behavior than rats raised in LP, in an open field test (OFT) and in elevated plus maze (EPM). Repeated comparisons showed that photoperiod effect was accentuated after 16 weeks of treatment. 24 weeks of treatment failed to induce any effect on emotionality in male rats. Our results indicate that changes in day length are associated with different levels of anxious-like behaviors; consistent with the conjecture that early life photoperiod may influence affective behavior in adult male rats.
\end{abstract}

Keywords: Photoperiod, Early Life Photoperiod, Anxiety-Like Behavior

\section{Introduction}

The seasonal change in day length (photoperiod) provides an indication of time-of-year; photoperiodic entrainment of an endogenous circadian rhythm in pineal melatonin secretion evokes changes in many seasonal adaptations, including reproduction and metabolism [1-3]. Not only are these traits influenced by photoperiod in mammals that respond reproductively to photoperiod, behavioral responses to photoperiod are also observed in mammals that do not respond reproductively to photoperiod, which has implications for developing animal models of human pathologies with a seasonal component, such as seasonal affective disorders and depression [4,5]. SAD is certainly the most well known of psychiatric phenomena occurring with a seasonal pattern and has gained increasing attention after its first description [6] and after the discovery that bright light treatment could inhibit melatonin secretion in humans [7] and alleviate symptoms $[8,9]$.

Recent research has now identified several rodent species that display changes in affective behaviors that are induced by changes in photoperiod. In reproductively photoperiodic rodents, exposure to short days induces changes in affective behaviors that are independent of changes in reproductive hormones. Exposure to short days increases anxiety-like and depressive-like responses in collared lemmings [10], and Siberian hamsters [11]. Animals that do not respond reproductively to photoperiod also display photoperiodic responses, Short-day exacerbation of depressive-like and anxiety-like behaviors have been reported in both nocturnal rodents [12-14], and diurnal rodents (e.g., Nile grass rats [15], and sand rats $[16,17])$.

In addition many adult diseases and disorders are influenced by the season of birth [18-20]. Some of these disorders can be modelled by early life differences in photoperiod. Although much research on seasonality in small mammals has focused on photoperiod manipulations in adults, early life photoperiod is also an important source of seasonal information and can establish individual's developmental trajectory by regulating somatic and reproductive development [1] and affective responses to day lengths later in life. Hamsters exposed to short days early in life have increased anxiety-and depressive- 
like responses as adults [21].

We designed the present study to further characterize the effect of photoperiod exposure at birth on anxiety like behavior in rat.

\section{Methods}

\subsection{Animals}

Breeding pairs were composed of adult male and female Wistar rats. Pairs were housed in polypropylene cages in a room illuminated for $12 \mathrm{~h}$ per day with fluorescent light (12L/12D; light onset at $0800 \mathrm{~h}$ ); Food and water were provided ad libitum; wood ship was available in the cage. Pairs were inspected daily for the presence of pups, and the day of birth was designated as day 0 where they were either transferred to a short (SP: 8L/16D) or long (LP: 16L/8D) photoperiod. The onset of the light phase occurred at the same clock time $(08.00 \mathrm{~h}$.) in all rooms. This lighting regime was set based on previous results [12-14], and according to day length in summer and winter. Pups $(\mathrm{n}=12)$ were weaned on day 20. From the age of 8 weeks, rats were submitted to different behavioral tests to quantify anxiety-like behaviors three times at interval of 8 weeks to evaluate the effect of such treatment

\subsection{Behavioural Testing}

All behavioural tests were conducted in a separate room. Animals were transferred to the test room an hour before testing to acclimatize rats. Behavioral testing separated by 24 hours were conducted during the diurnal phase, between 9:00 and 12:00 $\mathrm{h}$ in the order of their following description.

\subsubsection{Open Field Testing (OFT)}

The investigatory behavior was tested in a wooden made apparatus $(100 \mathrm{~cm} \times 100 \mathrm{~cm})$. As previously reported [22], it was enclosed with $40 \mathrm{~cm}$ high walls and placed under strong illumination (100 watt, $2 \mathrm{~m}$ above the apparatus). The area was divided into 25 squares $(20 \mathrm{~cm} \times$ $20 \mathrm{~cm}$ ), defined as nine central and sixteen peripheral squares. At the beginning of the 10-min test, the animal was placed in the centre of the apparatus and its behavior was videotaped for subsequent analysis. The device was cleaned after each individual rat session. The quantified parameters were the time spent in the center of the area (TCA) and the number of returns to the nine square central sections (NRC). Central perimeter residence time is used as a measure of anxiety [23]. The number of returns to the central area is also an indicator of the emotional reactivity [22]. The central area of a novel environment is anxiogenic and aversive and the behavioral inhibition appears therefore as an avoidance behavior towards the central zone of the open field [24].

\subsubsection{Elevated plus Maze (EPM)}

The EPM is an ethological model of anxiety in rodents provoked by the novelty and repulsion as a result of elevation and illumination of the maze [25]. This test is based on the creation of a conflict between the exploratory drive of the rat and its innate fear of open and exposed areas; it has been validated for the detection of emotional responses to anxiogenic and anxiolytic substances [26]. Thus, increased open-arm exploration indicates reduced anxiety-related behavior. The EPM consists of a wooden plus-shaped platform elevated $70 \mathrm{~cm}$ above the floor. Two of the opposing arms $(50 \mathrm{~cm} \times 10$ $\mathrm{cm})$ are closed by $40 \mathrm{~cm}$-high side and end walls, having an open roof. In order to avoid fall, the other two arms (open arms) were surrounded by $0.5 \mathrm{~cm}$ high edge, the four arms had at their intersection a central platform (10 $\mathrm{cm} \times 10 \mathrm{~cm}$ ) [23]. A 100-W lamp was placed exactly over the central platform. At the beginning of the test, the rats were placed on the central area of the maze facing an open arm. Behavior was video tapped during 5 min for time spent in each section of the maze and number of entries. Time spent in the arms and number of entries was used to compute time and entries ratio (open/closed) that were used for analysis. Decreased anxiety-like behavior is illustrated by a significant statistical increase of parameters in open arms (time and/or entries). Although total entries reflect the motor component of the exploratory activity [25].

\section{Statistics}

The results are expressed as mean \pm S.E.M. Main effects of photoperiod (LP, SP) and duration (Eight (8 W), sixteen (16 W), or twenty four ( $24 \mathrm{~W}$ ) weeks of photoperiod treatment), and interactions there of, on behavioral measures were assessed.

Data were subject to a repeated-measure analysis of variance with photoperiod treatment as variable and duration of treatment as factor, when appropriate, LSD post hoc was performed.

Mann-Whitney test was used to compare PL and SP groups in each period of treatment.

Differences were considered significant when $p<$ 0.05 .

\section{Results}

\subsection{Open Field Test}

\subsubsection{Time Spent in the Central Area (TCA) (Figure 1(a))}

The photoperiod affected significantly the total amount of time spent in center area (TCA) of the open field, this parameter was greater in rats exposed to $\operatorname{LP}\left(F_{(1,10)}=\right.$ $4.98 ; p=0.03)$. TCA also differed among duration $\left(F_{(2,20)}\right.$ 


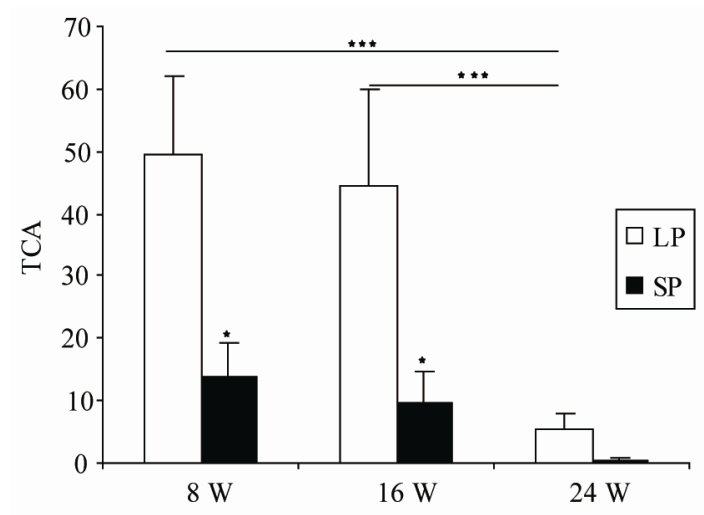

(a)

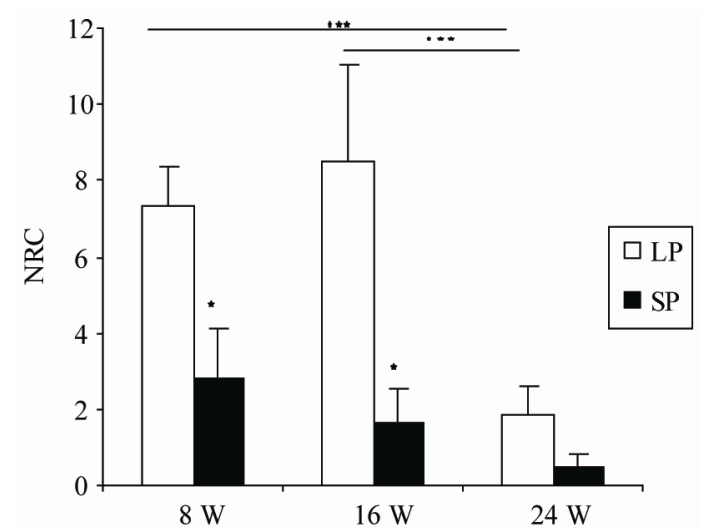

(b)

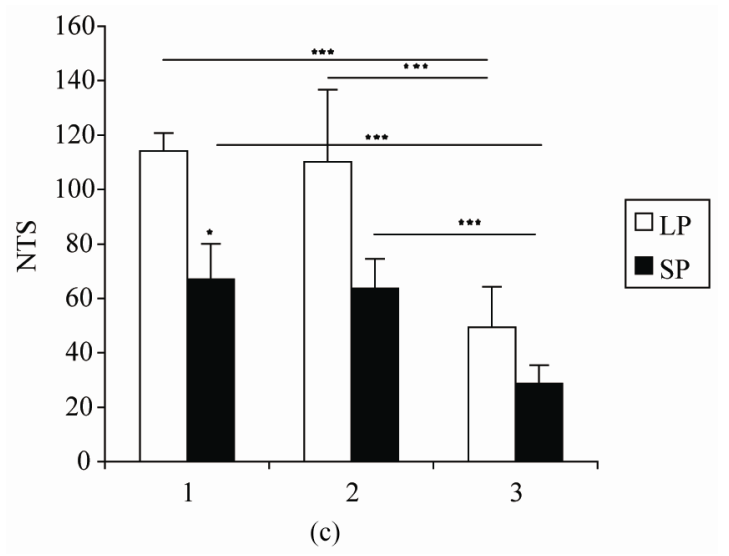

Figure 1. Mean (SEM) (a) Total amount time spent in the center of the open field (TCA), (b) number of return into center area of the arena in the open-field behaviour apparatus (NRC), and (c) Number of total squares crossed by male rats exposed to $L P$, or SP beginning at 0 day old and during $8(8 \mathrm{~W}), 16(16 \mathrm{~W})$ or $24(24 \mathrm{~W})$ weeks of treatment. Values at distal ends of horizontal bar differ, ${ }^{*} p<0.05,{ }^{* *} p<$ $0.01,{ }^{* * *} p<0.001$.

$=15.31, p=0)$. There was interaction between photoperiod and duration $\left(F_{(2,30)}=5.26, p=0.015\right)$.

In LP room, LSD test revealed that the time spent in the center area of OFT was greater in rats treated during $8(p=0)$ and 16 weeks $(p=0)$ in comparison with rats exposed to photoperiod regimen within 24 weeks.

In SP rats, Repeated comparisons did not show any significance differences between different groups (SP8/ SP16: $p=0.58$; SP8/SP24: $p=0.09$; SP16/SP24: $p=$ $0.28)$.

In each treatment duration, the statistical comparison scored that after 8 and 16 weeks of treatment, LP rats spent significantly more time in the central area of the OFT than did SP ones ( $p=0.02, p=0.04$ respectively). However, there was no significant difference between LP and SP rats after 24 weeks of treatment $(p=0.17)$.

\subsubsection{Number of Returns to the Center (NRC) (Figure 1(b))}

The number of returns to the central area differed among duration $\left(F_{(2,20)}=14.68, p=0\right)$ and photoperiod $\left(F_{(1.10)}=\right.$ $6.74 ; p=0.027)$. LP animals returned more often to the center area of the open field than did SP ones. There was an interaction between the two independent factors $\left(F_{(2,20)}\right.$ $=5.4 ; p=0.013$ )

LSD comparisons showed that, relative to LP24, LP8 and LP16 returned significantly more often to the center area ( $p=0.0001, p=0.00002$, respectively), no difference was noted between LP8 and LP16 ( $p=0.33)$.

In SP room, any significance differences between different groups was noted (SP8/SP16: $p=0.33$; SP8/SP24: $p=0.062 ; \mathrm{SP} 16 / \mathrm{SP} 24, p=0.33)$.

After 8 and 16 weeks of exposure to photoperiod regimen, LP rats returned significantly more often to the center than did SP ones ( $p=0.03, p=0.04$ respectively), whereas in $24 \mathrm{~W}$ rats, this parameter was not affected by photoperiod $(p=0.2)$.

\subsubsection{Locomotor Activity (NTS) (Figure 1(c))}

The number of total squares (NTS) visited in the open field was not significantly affected by photoperiod, $\left(F_{(1,10)}\right.$ = 4.15, $p=0.068$ ).

This parameter was significantly affected by duration $\left(F_{(2,20)}=25.58, p=0\right)$. There was no interaction between photoperiod and duration $\left(F_{(2,20)}=1.76, p=0.19\right)$.

Fisher's LSD test has shown that in LP room the number of total squares visited was greater in rats treated during 8 and 16 weeks than those treated during 24 weeks $(p=0)$. LP8 and LP16 did not show any difference for this parameter ( $p=0.73)$.

In SP groups, LSD comparisons revealed that SP8 and SP16 exhibited more locomotor activity than SP24 ( $p=$ 0.003; $p=0.006$ respectively), SP8 and SP16 did not show any difference for this parameter $(p=0.77)$.

After 8 weeks of treatment, rats heberged in LP exhibited more locomotor activity than SP rats $(p=0.02)$. However, this parameter was not affected by photoperiod after 16 and 24 weeks of treatment $(p=0.30 ; p=0.48$ 
respectively).

\subsection{Elevated plus Maze}

\subsubsection{Open/Closed Entries Ratio (Figure 2(a))}

This parameter was not affected by photoperiod $\left(F_{(1,10)}=\right.$ $0.02, p=0.89$ ) but was significantly affected by duration $\left(F_{(2,20)}=4.15, p=0.03\right)$. There was no interaction between duration and photoperiod $\left(F_{(2,20)}=2.7, p=0.09\right)$.

Post-hoc comparisons revealed that LP16 and LP24 visited significantly more often the open arms than did LP8 ( $p=0.025, p=0.02$ respectively). LP16 and LP24 did not differed among this parameter $(p=0.92)$.

In SP groups, $24 \mathrm{~W}$ visited significantly more often the open arms than did SP16 ones $(p=0.03)$. No difference between other groups was noted (SP8/SP16: $p=$ 0.43; SP8/SP24: $p=0.14$ ).

After 8 and 24 weeks of treatment, no differences was noted between LP and SP rats ( $p=0.18, p=0.31$, respectively). Whereas, after exposure to photoperiod regimen during 16 weeks, LP rats visited significantly more often the exposed arms than SP ones ( $p=0.04)$.

\subsubsection{Open/Closed Time Ratio (Figure 2(b))}

No main effect related to photoperiod was evident in amount of time spent exploring exposed arms $\left(F_{(1,10)}=\right.$ $0.11, p=0.74)$. However duration affected significantly this parameter $\left(F_{(2,20)}=7.33 ; p=0.004\right)$. An interaction between photoperiod and duration was noted $\left(F_{(2,20)}=\right.$ 15.96, $p=0$ ).

In LP rats, Post-hoc comparisons revealed that LP16 spent more time in open arms relative to both LP8 $(p=0)$ and LP24 ( $p=0.0001)$. LP8 did not differ from LP24 for this parameter $(p=0.25)$.

In SP groups, SP24 spent significantly more time in exposed arms than SP8 $(p=0.04)$ and SP16 $(p=0.015)$. SP8 and SP16 behaved comparably for this parameter $(p$ $=0.62$ ).

In $8 \mathrm{~W}$ and $24 \mathrm{~W}$ no significant difference was observed between LP and SP rats $(8 \mathrm{~W}: p=0.21 ; 24 \mathrm{~W}: p$ $=0.13$ ). Whereas, after 16 weeks, LP rats spent significantly more time in open arms than did SP ones $(p=$ $0.01)$.

\subsubsection{Total Entries in Arms (TEA) (Figure 2(c))}

No main effect related to photoperiod was observed in the total entries in arms. $\left(F_{(1,10)}=1, p=0.33\right)$, whereas duration affected significantly this parameter $\left(F_{(2,20)}=5.4\right.$, $p=0.013)$. There was interaction between photoperiod and duration in the total entries in arms $\left(F_{(2,20)}=5.98, p=\right.$ 0.009 ).

After exposure to LP, LP16 visited a greater number of arms than LP8 $(p=0.003)$ and LP24 $(p=0.0002)$. However, LP8 and LP24 did not differ for this parameter $(p>0.55)$.

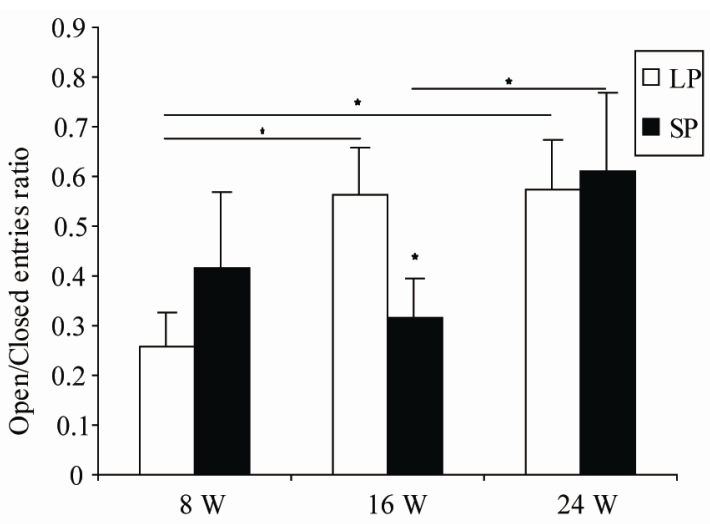

(a)

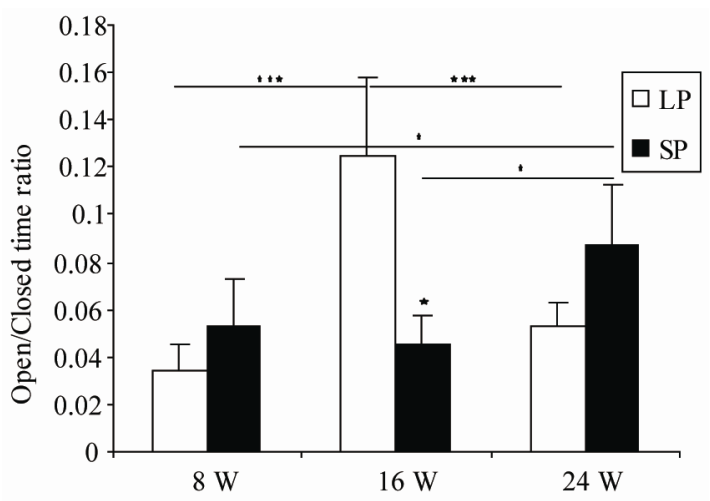

(b)

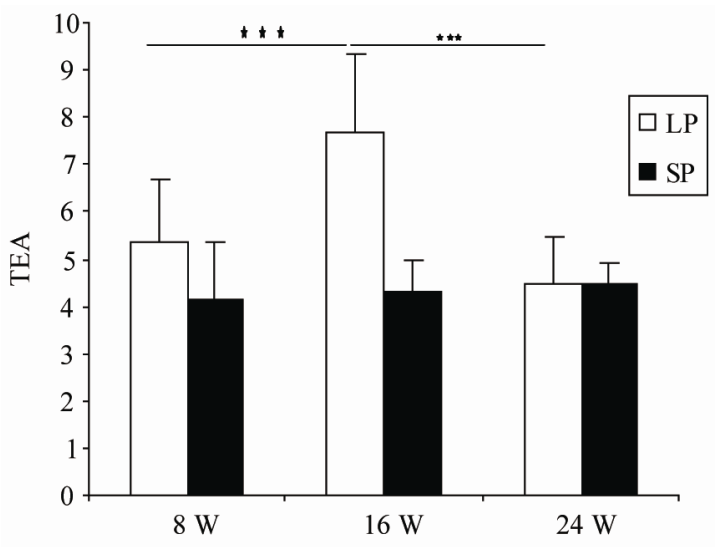

(c)

Figure 2. Mean (SEM) (a) number of entries in the two exposed arms of elevated plus maze (EOA), (b) total amount of time spent exploring these arms (TOA) and (c) total number of arm entries (TEA) scored by male rats exposed to LP, or SP beginning at 0 day old and during $8(8 \mathrm{~W}), 16$ $(16 \mathrm{~W})$ or $24(24 \mathrm{~W})$ weeks of treatment. Values at distal ends of horizontal bar differ, ${ }^{*} p<0.05,{ }^{* *} p<0.01,{ }^{* * *} p<$ 0.001.

In SP room, no significant difference was noted between different groups (SP8/SP16: $p>0.39$; SP8/SP24: $p$ $>0.061$; SP16/SP24: $p>0.28$ ).

No difference was noted between LP and SP rats in 
entries in arms whatever the duration treatment $(8 \mathrm{~W}, p=$ 0.48 ; $16 \mathrm{~W}, p=0.13 ; 24 \mathrm{~W}, p=0.48$ ).

\section{Discussion}

The goal of these experiments was to elucidate the effect of exposure to different photoperiods at birth on affective behavior in male rats. Rats exposed to SP during 8 and 16 weeks exhibited higher level of anxious-like behaviour than rats raised in LP as assessed by the NRC and TCA in Open Field Test. However, this photoperiod effect disappears after 24 weeks of treatment.

Repeated comparisons revealed that rats treated during 8 and 16 weeks did not show any difference in LP or SP room. After 24 weeks of LP treatment, the rats exploratory behaviour in the center of the open field decreased significantly relative to 8 and 16 weeks of treatment. However, performance in the center of the paradigm in rats exposed to SP increased in $24 \mathrm{~W}$ in comparison with SP8 and SP16.

In EPM, after 16 weeks of treatment, SP rats showed higher anxiety-like behaviour in this paradigm as measured by number of entries into and time spent in exposed arms than did LP rats. While, Rats treated during 8 and 24 weeks did not showed any significant difference between LP and SP rooms for these parameters.

LP16 rats exhibited higher exploratory behaviour in exposed arms potentially aversive than LP8 and LP24 ones. In SP room, SP16 showed higher anxiety-like behaviour than SP8 and SP24 rats which behaved similarly in this room.

Thus, only 16 weeks of treatment at birth was able to induce an effect on emotionality in EPM. 8 weeks were insufficient to induce an effect in EPM in rats treated at birth age.

Similarly to results in OFT, 24 weeks was ineffective in EPM measures. Anxiety-like behaviour decreased in SP and increased in LP in comparison with 16 weeks of treatment.Thus 24 weeks will be a photorefractory period for affective behaviour as it is for reproductive behaviour in hamsters [27-29]. This would imply that the Wistar rat had preserved neuroendocrine components that regulate photoperiodic response as reported in several researchs [30-34].

Together, these results indicate that early life photoperiod is sufficient to induce an affective behavior in rat in a duration dependent manner.

Early life photoperiod is also an important source of seasonal information.Young photoperiodic animals are exceptionally responsive to day length information; exposure to short days increases anxiety-like and depressive-like responses in collared lemmings [10] and Siberian hamsters [11,21]. These photoperiodic behavioral changes develop early during short-day exposure [11] and can persist after maximal gonadal regression [10,21] supporting the idea that the influence of photoperiod on affective behavior can be independent of circulating gonadal steroids. These affective responses to short days are unambiguously linked directly to pineal melatonin secretion duration [17], which may model the extended duration of melatonin secretion observed in humans with seasonal affective disorder [35].

In addition to the seasonally variable phenotypic changes, photoperiod history can also affect adult phenotype. Late gestation and birth in short days, in both northern and southern hemispheres, is associated with increased prevalence of schizoaffective disorder, autism, and major depression in the adult population $[18,19]$. Perinatal photoperiod conditions can organize adult affective behaviors and can interact with postweaning pho- toperiod conditions to regulate the expression of affective behaviors in adults [11,21].

Our results demonstrate that male rat can respond emotionally to photoperiod soon after birth and this response is exposure duration dependent. This result supports the photoperiod hypothesis in SAD etiology determination.

Day length is an unambiguous environmental signal. This signal can be used in the lab to explore the mechanisms by which the environment act to impart phenotype. Investigation of how this one factor acts to affect affective behavior may contribute to our understanding of clinical disorders and other pathologies in a translational setting.

\section{REFERENCES}

[1] F. H. Bronson, "Mammalian Reproductive Biology," University of Chicago Press, Chicago, 1989.

[2] B. D. Goldman, "Mammalian Photoperiodic System: Formal Properties and Neuroendocrine Mechanisms of PhotoPeriodic Time Measurement,” Journal of Biological Rhythms, Vol. 16, 2001, pp. 283-301. doi:10.1177/074873001129001980

[3] B. J. Prendergast, R. J. Nelson and I. Zucker, "Mammalian Seasonal Rhythms: Behavior and Neuroendocrine Substrates,” In: D. W. Pfaff, Ed., Hormones, Brain and Behavior, Academic Press, San Diego, 2002.

[4] J. L. Workman and R. J. Nelson, "Potential Animal Models of Seasonal Affective Disorder," Neuroscience \& Biobehavioral Reviews, Vol. 35, No. 3, 2010, pp. 669-679.

[5] N. Sinitskaya, C. Schuster-Klein, B. Guardiola-Lemaitre, S. Gourmelen, P. Pévet and E. Challet, "Short Day-Length Increases Sucrose Consumption and Adiposity in Rats Fed a High-Fat Diet,” Psychoneuroendocrinology, Vol. 33, No. 9, 2008, pp. 1269-1278.

[6] N. E. Rosenthal, D. A. Sack, J. C. Gillin, A. J. Lewy, F. K. Goodwin, K. Dakahashi, P. S. Mueller, D. A. Newsome and T. A. Wehr, "Seasonal Affective Disorder: A Descrip- 
tion of the Syndrome and Preliminary Findings with Light Therapy," Archives of General Psychiatry, Vol. 41, No. 1, 1984, pp. 72-80.

[7] A. J. Lewy, T. A Wehr, F. K. Goodwin, D. A. Newsome and S. P. Markey, "Light Suppresses Melatonin Secretion in Humans,” Science, Vol. 210, No. 4475, 1980, pp. 12671269.

[8] N. E. Rosenthal, D. A. Sack, C. J. Carpenter, B. L. Parry, W. B. Mendelson and T. A. Wehr, "Antidepressant Effects of Light in Seasonal Affective Disorder," American Journal of Psychiatry, Vol. 142, No. 2, 1985, pp. 163-170.

[9] T.A. Wehr, F. M. Jacobsen, D. A. Sack, J. Arendt, L. Tamarkin and N. E. Rosenthal, "Phototherapy of Seasonal Affective Disorder. Time of Day and Suppression of Melatonin Are Not Critical for Antidepressant Effects," Archives of General Psychiatry, Vol. 43, No. 9, 1986, pp. 870-875.

[10] Z. M. Weil, S. L. Bowers and R. J. Nelson, "Photoperiod Alters Affective Responses in Collared Lemmings," Behavioural Brain Research, Vol. 179, No. 2, 2007, pp. 305309.

[11] B. J. Prendergast and R. J. Nelson, “Affective Responses to Changes in Day Length in Siberian Hamsters (Phodopus sungorus)," Psychoneuroendocrinology, Vol. 30, No. 5, 2005, pp. 438-452.

[12] N. Benabid, A. Mesfioui and A. Ouichou, "Effects of Photoperiod Regimen on Emotional Behaviour in Two Tests for Anxiolytic Activity in Wistar Rat," Brain Research Bulletin, Vol. 75, No. 1, 2008, pp. 55-59. doi:10.1016/j.brainresbull.2007.07.016

[13] M. Molina-Hernandez and P. Tellez-Alcantara, "LongPhoto-Period Regimen May Produce Antidepressant Actions in the Male Rat," Progress in Neuro-Psychopharmacology \& Biological Psychiatry, Vol. 24, No. 1, 2000, pp. 105-116. doi:10.1016/S0278-5846(99)00084-6

[14] B. J. Prendergast and L. M. Kay, "Affective and Adrenocorticotrophic Responses to Photoperiod in Wistar Rats," Journal of Neuroendocrinology, Vol. 20, No. 2, 2008, pp. 261-267. doi:10.1111/j.1365-2826.2007.01633.x

[15] T. Ashkenazy-Frolinger, N. Kronfeld-Schor, J. Juetten and H. Einat, "It Is Darkness and Not Light: Depression-Like Behaviors of Diurnal Unstriped Nile Grass Rats Maintained Under a Short Photoperiod Schedule," Journal of Neuroscience Methods, Vol. 186, No. 2, 2010, pp 165-170. doi:10.1016/j.jneumeth.2009.11.013

[16] T. Ashkenazy, H. Einat and N. Kronfeld-Schor, "Effects of Bright Light Treatment on Depression- and Anxiety-Like Behaviors of Diurnal Rodents Maintained on A Short Daylight Schedule,” Behavioural Brain Research, Vol. 201, No. 2, 2009, pp. 343-346. doi:10.1016/j.bbr.2009.03.005

[17] T. Ashkenazy, H. Einat and N. Kronfeld-Schor, "We Are in the Dark Here: Induction of Depression- and Anxiety-Like Behaviours in the Diurnal Fat Sand Rat, by Short Daylight or Melatonin Injections,” The International Journal of Neuropsychopharmacology, Vol. 12, No. 1, 2009, pp. 83-93. doi:10.1017/S1461145708009115
[18] E. H. Hare, "Variations in the Seasonal Distribution of Births of Psychotic Patients in England and Wales," British Journal of Psychiatry, Vol. 132, 1978, pp. 155-158. doi:10.1192/bjp.132.2.155

[19] E. F. Torrey, J. Miller, R. Rawlings and R. H. Yolken, "Seasonality of Births in Schizophrenia and Bipolar Disorder: A Review of the Literature," Schizophrenia Research, Vol. 28, No. 1, 1997, pp. 1-38. doi:10.1016/S0920-9964(97)00092-3

[20] P. Dome, B. Kapitany, G. Ignits and Z. Rihmer, "Season of Birth Is Significantly Associated with the Risk of Completed Suicide,” Biological Psychiatry, Vol. 68, No. 2, 2010, pp. 148-155.doi:10.1016/j.biopsych.2010.03.005

[21] L. M. Pyter and R. J. Nelson, "Enduring Effects of Photoperiod on Affective Behaviors in Siberian Hamsters (Phodopus Sungorus),” Behavioral Neuroscience, Vol. 120, No. 1, 2006, pp. 125-134. doi:10.1037/0735-7044.120.1.125

[22] M. Durand, O. Berton, S. Aguere, L. Edno, I. Combourieu, P. Mormède and F. Chaouloff, "Effects of Repeated Fluoxetine on Anxiety-Related Behaviour," Journal of Neuropharmacology, Vol. 38, 1999, pp. 893-907.

[23] A. Nasselo, G. Machado and C. Bastos, "Sudden Darkness Induces a High Activity-Low Anxiety State in Male and Female Rats,” Physiology \& Behavior, Vol. 63, No. 3, 1998, pp. 451-454.

[24] L. Meyer, J. Caston and A. G. Mensah-Nyagan, "Seasonal Variation of the Impact of a Stressful Procedure on Open Field Behaviour and Blood Corticosterone in Laboratory Mice,” Behavioural Brain Research, Vol. 167, No. 2, 2006, pp. 342-348. doi:10.1016/j.bbr.2005.09.023

[25] F. Clénet, E. Bouyon, M. Hascoët and M. Bourin, "Light/ Dark Cycle Manipulation Influences Mice Behavior in the Elevated Plus Maze,” Behavioural Brain Research, Vol. 166, 2006, pp.140-149.

[26] S. Pellow, P. Chopin, S. E. File and M. Briley, "Validation of Open: Closed Arms Entries in an Elevated Plus-Maze as a Measure of Anxiety in the Rat," Journal of Neuroscience Methods, Vol. 14, No. 3, 1985, pp. 149-167. doi:10.1016/0165-0270(85)90031-7

[27] B. Hoffmann, "Aspects on the Formation and Detection of Tissue Levels of Anabolic Steroids in Domestic Animals,” Journal of Steroid Biochemistry, Vol. 11, No. 1, 1979, pp. 919-922. doi:10.1016/0022-4731(79)90030-X

[28] P. Pévet, "The Role of the Pineal Gland in the Photoperiodic Control of Reproduction in Different Hamster Species,” Reproduction, Nutrition and Development, Vol. 28, No. 2B, 1988, pp. 443-458. doi:10.1051/rnd:19880310

[29] A. Lerchl and S. Schlatt, "Influence of Photoperiod on Pineal Melatonin Synthesis, Fur Color, Body Weight, and Reproductive Function in the Female Djungarian Hamster, Phodopus Sungorus,” Neuroendocrinology, Vol. 57, No. 2, 1993, pp. 359-364.

[30] J. Hoffmann, C. Kordon and J. Benoit, "Effect of Different Photoperiods and Blinding on Ovarian and Testicular Functions in Normal and Testosterone Treated Rats," General and Comparative Endocrinology, Vol. 10, No. 1, 1968, pp. 109-118. 
[31] R. J. Nelson, C. A. Moffatt and B. D. Goldman, "Reproductive and Nonreproductive Responsiveness to Photoperiod in Laboratory Rats,” Journal of Pineal Research, Vol. 17, No. 3, 1994, pp. 123-131. doi:10.1111/j.1600-079X.1994.tb00123.x

[32] R. J. Reiter, J. C. Hoffman and P. H. Rubin, "Pineal Gland: Influence on Gonads of Male Rats Treated with Androgen 3 Days after Birth,” Science, Vol. 160, No. 826, 1968, pp. 420-421.

[33] J. Vanecek and H. Illnerova, "Effect of Photoperiod on the Growth of Reproductive Organs and on Pineal N-Cetyl-Transferase Rhythm in Male Rats Treated Neo- natally with Testosterone Propionate," Biology of Reproduction, Vol. 27, 1982, pp. 517-522.

doi:10.1095/biolreprod27.3.517

[34] E. P. Wallen and F. W. Turek, "Photoperiodicity in the Male Albino Laboratory Rat," Nature, Vol. 289, No. 5796, 1981, pp. 402- 404.

[35] T. A. Wehr, W. C. Duncan Jr., L. Sher, D. Aeschbach, P. J. Schwartz, E. H. Turner, T. T. Postolache and N. E. Rosenthal, "A Circadian Signal of Change of Season in Patients with Seasonal Affective Disorder," Archives of General Psychiatry, Vol. 58, No. 12, 2001, pp. 1108-1114. doi:10.1001/archpsyc.58.12.1108 